\title{
Professor Seung-Yull Cho (1943-2019)
}

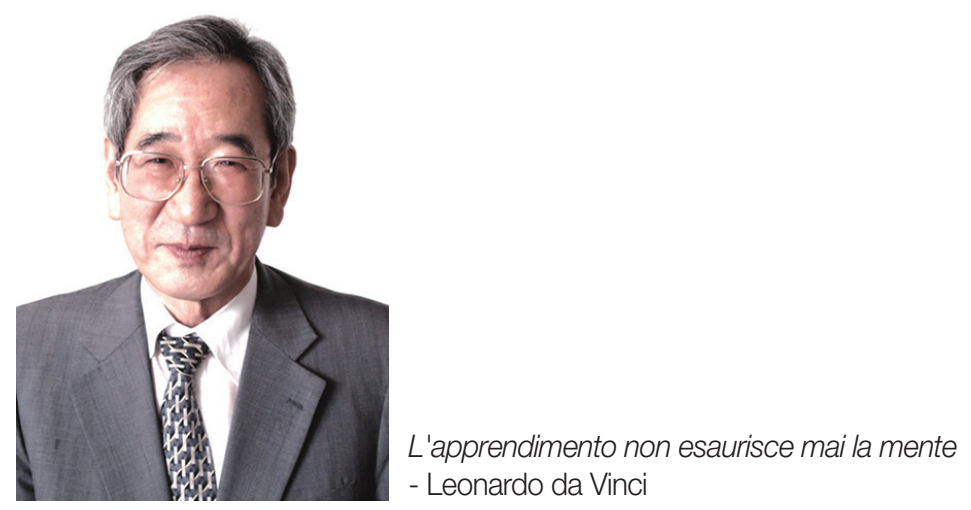

Korean parasitology and the academic society of the Republic of Korea lost an outstanding person and ambassador for medical science with the decease of Professor Seung-Yull Cho in Seoul on 27 January 2019. A world-class parasitologist, a leader of academic society, and an organizer and supporter of editors for medical publications, contributions by him were enormous and diverse in a lifetime devoted to medical science.

He was born on 16 November 1943 in Seoul, the city where he spent almost all his lifetime. He graduated from Seoul National University, College of Medicine. He was trained at the Department of Parasitology, Seoul National University, College of Medicine. As an enthusiastic and energetic student under mentorship from Professor Byong-Seol Seo, he was particularly interested in control of intestinal nematode infections, which had been highly prevalent in Korea at that time. He did pioneering works on mass chemotherapy and evaluation of anthelminthic efficacy and epidemiological studies including infection dynamics. He proposed a model for mass chemotherapeutic control of filariasis, ascariasis, and enterobiasis. His studies provided information about general patterns in the distribution of geohelminths and reinfection dynamics. In 1973 he defended a dissertation on "Study of the quantitative evaluation of reinfection of Ascaris lumbricoides", for which he was conferred a doctoral degree. This article brought him to be a winner of an academic honor on 1978 by the Korean Society for Parasitology (currently The Korean Society for Parasitology and Tropical Medicine).

During 1974-2009, he served as a professor of parasitology in several academic institutions, which included Seoul National University (1974-1981), Chung-Ang University (19811995), The Catholic University of Korea (1995-1998), and Sungkyunkwan University (1998-2009) more than 35 years. After retirement, he attended in Gacheon University as an invited professor (2009-2018).

Exposure and immersion in the new environment were critical to pursue a career path as a medical scientist. The early 1980s were an exciting time of expansion of his scientific excursion. He built up strong conceptual foundation in hostparasite interactions mediated by antibody responses and focused his interests on serological diagnosis of tissue invading helminth parasites, such as Taenia solium metacestode, Paragonimus westermani and the sparganum. These parasites still trigger critical food-borne helminthiases associated with disability-adjusted life years and sometimes invoke life-threatening illness globally. He applied serodiagnostic test to detect specific antibody levels in biomaterials derived from these patients and enhanced serodiagnostic feasibility by adapting enzyme-linked immunosorbent assay system. He extended his researches in analyzing relationships between clinicopathological changes and alteration of serological profile during the 
course of helminthic infections and between serological responsiveness and radiological characteristics. The diagnosis of parasitic diseases, especially those involving the central nervous system, could be substantially improved. He was strict to himself, but generous to other people. He was renowned for his patience, passions, and critical comments of the works of colleagues during collaboration with other scientists. He always sought "beyond horizon". Through this enthusiastic effort, he published approximately 300 scientific papers in the prestigious journals, most of which are experimental in nature.

He also dedicated himself as a leader of academic community, philologist of medical terminology, editor of medical publications, mentor for young scientists in developing careers, and supporter for medical editors. He was a president of Korean Society for Parasitology (1988-1989) and Korean Association of Medical Editors (2002-2005). He was elected as a president of National Academy of Medical Science of Korea (2010-2013). He had been an excellent editor of Korean Journal of Parasitology (1976-1987) and Journal of Korean Medical Sciences (1997-2006) over 20 years. As a result of his dedi- cation he was an awardee of Korean Academy of Medical Sciences on 2015 (KAMS Honor in Medicine Award).

Professor Seung-Yull Cho is clearly one of the most talented parasitologists of Korea and has been widely respected for his contributions in the field of medical science, not only in the field of parasitology. His innovative works in parasitology and journal editing through his lifetime will never be forgotten. Colleagues remember him as an enthusiastic helminthologist, great organizer and administrator, and supporter.

We have lost a dear scholar, mentor, and friend. Korea has lost a true ambassador of parasitology and medical science, who has spent his life in the virtue of an ideal spirit. With the deepest condolence and sorrowful love of the rest, we burn incense. Rest in peace in endless divine afterworld.

\section{Yoon Kong}

Department of Molecular Parasitology, Sungkyunkwan University School of Medicine, Suwon 16419, Korea.

kongy@skku.edu 